\title{
Clinical utility gene card for: Phosphomannomutase 2 deficiency
}

\author{
Jaak Jaeken $^{\star, 1}$, Dirk Lefeber ${ }^{2}$ and Gert Matthijs ${ }^{3}$ \\ European Journal of Human Genetics (2014) 22, doi:10.1038/ejhg.2013.298; published online 15 January 2014
}

\section{DISEASE CHARACTERISTICS}

1.1 Name of the disease (synonyms)

Phosphomannomutase 2 deficiency, PMM2-CDG, CDG-Ia, Jaeken disease.

\subsection{OMIM\# of the disease \\ 212065.}

1.3 Name of the analysed genes or DNA/chromosome segments PMM2.

\subsection{OMIM\# of the gene(s)}

601785 .

\subsection{Mutational spectrum}

Some 107 mutations have been reported, including 84 missense mutations, 5 nonsense mutations, 7 frameshift mutations, 10 splicing defects, and 1 complete loss of exon 8 due to a deletion mediated by an Alu retrotransposition ${ }^{1,2}$ (www.lovd.nl/PMM2). The c.422G $>$ A (p.R141H) is the most frequent mutation, present in $75 \%$ of patients of Caucasian origin. No patient homozygous for this mutation has been reported, because this severe mutation is not compatible with life in the homozygous state. ${ }^{3-5}$ The standard reference sequence indicating reported variants (ENSG00000140650) and a reference for exon numbering (ENST00000268261) can be found at www.ensemble.org.

\subsection{Analytical methods}

Sanger sequencing of the eight exons and flanking intronic sequences of the PMM2 gene (NCBI reference sequence NM_000303.2).

\subsection{Analytical validation}

Sanger sequencing identifies mutations in $>99 \%$ of patients with an enzymatically confirmed PMM deficiency. Deep intronic mutations and deletions would be missed using this approach. ${ }^{6}$

1.8 Estimated frequency of the disease (incidence at birth ('birth prevalence') or population prevalence. If known to be variable between ethnic groups, please report):

The disease frequency has been calculated at 1/20000 in the Danish and the Dutch populations. ${ }^{7}$ Beware that the carrier frequency of the p.R141H is as high as 1 in 60 in some populations. ${ }^{7}$

\subsection{Diagnostic setting}

\begin{tabular}{lll}
\hline & Yes & No \\
A. (Differential) diagnostics & $\Downarrow$ & $\square$ \\
B. Predictive testing & $\bigotimes$ & $\square$ \\
C. Risk assessment in relatives & $\bigotimes$ & $\square$ \\
D. Prenatal & $\bigotimes$ & $\square$ \\
\hline
\end{tabular}

\section{Comment:}

PMM2-CDG is an autosomal recessive disorder with a very broad clinical spectrum ranging from prenatal death to a near normal presentation. ${ }^{8}$ All organs can be affected but there is always a predominant neurological involvement. In the typical presentation with fat pads and inverted nipples, the diagnosis can be clinically suspected. The biochemical screening test 'par excellence' is serum transferrin isoelectrofocusing, but this test can be normal in infants in the first 4-6 weeks of life and can be (near)-normal in mildly affected individuals and adults. The next diagnostic step is enzymatic analysis of phosphomannomutase in leukocytes or fibroblasts. Intermediate values (including values in the normal range) in fibroblasts may mask the diagnosis. Therefore, measurement of phosphomannomutase activity in leukocytes is preferable. ${ }^{9}$ The diagnosis has to be confirmed by mutation analysis of PMM2. This will permit heterozygote detection in the family and prenatal diagnosis. It has to be stressed that there is a higher than one in four risk in these families (closer to one in three). This needs to be considered in the genetic counselling. ${ }^{10}$

\section{TEST CHARACTERISTICS}

\begin{tabular}{|c|c|c|c|c|}
\hline & \multicolumn{2}{|c|}{ Genotype or disease } & \multirow{2}{*}{$\begin{array}{l}\text { A: True positives } \\
\text { B: False positives }\end{array}$} & \multirow{2}{*}{$\begin{array}{l}\text { C: False negative } \\
\text { D: True negative }\end{array}$} \\
\hline & Present & Absent & & \\
\hline \multicolumn{5}{|l|}{ Test } \\
\hline \multirow[t]{2}{*}{ Positive } & $A$ & B & Sensitivity: & $A /(A+C)$ \\
\hline & & & Specificity: & $D /(D+B)$ \\
\hline \multirow[t]{2}{*}{ Negative } & C & $D$ & Pos. predict. value: & $A /(A+B)$ \\
\hline & & & Neg. predict. value: & $D /(C+D)$ \\
\hline
\end{tabular}

${ }^{1}$ Centre for Metabolic Disease, University Hospital Gasthuisberg, KU Leuven, Leuven, Belgium; ²Department of Neurology, Laboratory of Genetic, Endocrine and Metabolic Disease, Institute for Genetic and Metabolic Disease, Radboud University Nijmegen Medical Centre, Nijmegen, The Netherlands; ${ }^{3}$ Centre for Human Genetics, KU Leuven, Leuven, Belgium

*Correspondence: Professor J Jaeken, Centre for Metabolic Disease, University Hospital Gasthuisberg, KU Leuven, Herestraat 49, Leuven BE 3000, Belgium. Tel: +32 16 343820; Fax: +32 16 343842; E-mail: jaak.jaeken@uzleuven.be 
2.1 Analytical sensitivity

(proportion of positive tests if the genotype is present)

Close to $100 \%$ when using the serum transferrin isoelectrofocusing test. This test can be normal in very mild cases and in adults. ${ }^{11}$

\subsection{Analytical specificity}

(proportion of negative tests if the genotype is not present)

Close to $100 \%$ when using the serum transferrin isoelectrofocusing test. This test can be positive in secondary glycosylation disorders such as galactosemia and hereditary fructose intolerance. ${ }^{12}$

\subsection{Clinical sensitivity}

(proportion of positive tests if the disease is present)

The clinical sensitivity can be dependent on variable factors such as age or family history. In such cases, a general statement should be given, even if a quantification can only be made case by case.

Close to $100 \%$.

\subsection{Clinical specificity}

(proportion of negative tests if the disease is not present)

The clinical specificity can be dependent on variable factors such as age or family history. In such cases, a general statement should be given, even if a quantification can only be made case by case.

Close to $100 \%$.

2.5 Positive clinical predictive value

(life-time risk to develop the disease if the test is positive)

$100 \%$, based on mutation analysis.

2.6 Negative clinical predictive value

(probability of not developing the disease if the test is negative) Assume an increased risk based on family history for a non-affected person. Allelic and locus heterogeneity may need to be considered.

Index case in that family had been tested:

$100 \%$.

Index case in that family had not been tested:

$100 \%$.

\section{CLINICAL UTILITY}

3.1 (Differential) diagnostics: the tested person is clinically affected (To be answered if in 1.9 'A' was marked)

\subsubsection{Can a diagnosis be made other than through a genetic test?}

No $\square$ (continue with 3.1.4)

Yes $\otimes$

Clinically
Imaging
Endoscopy
Biochemistry
Electrophysiology
Other (please describe)

Other (please describe) PMM enzymatic activity in leukocytes/fibroblasts. ${ }^{13}$

3.1.2 Describe the burden of alternative diagnostic methods to the patient

In many patients the clinical picture is suggestive. The blood sampling for the serum transferrin isoelectrofocusing screening test and that for the measurement of PMM enzymatic activity in leukocytes or fibroblasts and PMM2 gene analysis are a minor burden to the patient.
3.1.3 How is the cost effectiveness of alternative diagnostic methods to be judged?

The enzymatic test is only available in a limited number of laboratories (see orpha.net for the list).

3.1.4 Will disease management be influenced by the result of a genetic test?

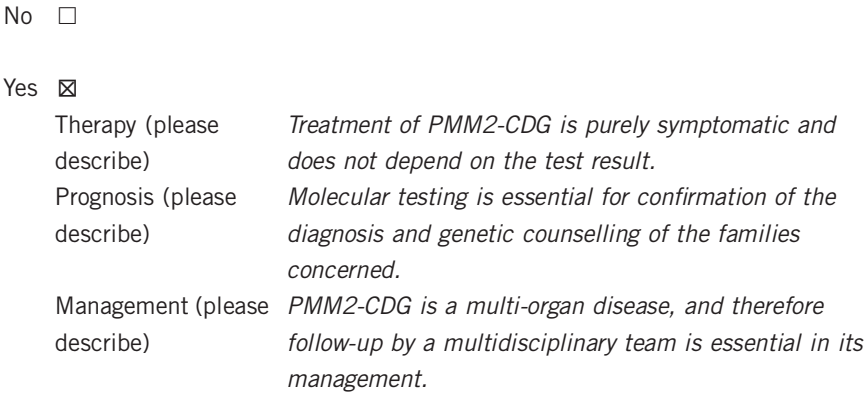

3.2 Predictive setting: the tested person is clinically unaffected but carries an increased risk based on family history

(To be answered if in 1.9 'B' was marked)

3.2.1 Will the result of a genetic test influence lifestyle and prevention?

If the test result is positive (please describe)

Not applicable.

If the test result is negative (please describe):

Not applicable.

3.2.2 Which options in view of lifestyle and prevention does a person at-risk have if no genetic test has been done (please describe)? Not applicable.

3.3 Genetic risk assessment in family members of a diseased person (To be answered if in 1.9 ' $\mathrm{C}$ ' was marked)

3.3.1 Does the result of a genetic test resolve the genetic situation in that family?

Usually yes, by testing the potential heterozygous persons (carriers) in the family.

3.3.2 Can a genetic test in the index patient save genetic or other tests in family members?

No.

3.3.3 Does a positive genetic test result in the index patient enable a predictive test in a family member?

Not applicable.

3.4 Prenatal diagnosis

(To be answered if in 1.9 ' $\mathrm{D}$ ' was marked)

3.4.1 Does a positive genetic test result in the index patient enable a prenatal diagnosis?

Yes.

Prenatal diagnosis should be performed by molecular or enzymatic analysis; foetal transferrin isoelectrofocusing leads to false results. ${ }^{14}$ Of course, mutation analysis in pregnancy is only reliable when the mutation in the index patient is available. 


\section{IF APPLICABLE, FURTHER CONSEQUENCES OF TESTING}

Please assume that the result of a genetic test has no immediate medical consequences. Is there any evidence that a genetic test is nevertheless useful for the patient or his/her relatives? (please describe).

Knowledge of the diagnosis will stop unnecessary further investigations, and will help the parents in the process of accepting the disease although no curative treatment is available.

\section{CONFLICT OF INTEREST}

The authors declare no conflict of interest.

\section{ACKNOWLEDGEMENTS}

This work was supported by EuroGentest2 (Unit 2: 'Genetic testing as part of health care'), a Coordination Action under FP7 (Grant Agreement Number 261469) and the European Society of Human Genetics.

1 Haeuptle MA, Hennet T: Congenital disorders of glycosylation: an update on defects affecting the biosynthesis of dolichol-linked oligosaccharides. Hum Mutat 2009; 30: 1-14.

2 Pérez B, Briones P, Quelhas D et al: The molecular landscape of phosphomannose mutase deficiency in Iberian peninsula: identification of 15 population-specific mutations. JIMD Rep 2011; 1: 117-123.

3 Kjaergaard S, Skovby F, Schwartz M: Absence of homozygosity for predominant mutations in Danish patients with carbohydrate-deficient glycoprotein syndrome type 1. Eur J Hum Genet 1998; 6: 331-336.
4 Matthijs G, Schollen E, Van Schaftingen E, Cassiman J-J, Jaeken J: Lack of homozygotes for the most frequent disease allele in carbohydrate-deficient glycoprotein syndrome type Ia. Am J Hum Genet 1998; 62: 542-550.

5 Pirard M, Matthijs G, Heykants L et al: Effect of mutations found in patients with carbohydrate-deficient glycoprotein syndrome type la on the activity of phosphomannomutase 2. FEBS Lett 1999; 452: 319-322.

6 Schollen E, Keldermans L, Foulquier $\mathrm{F}$ et al: Characterization of two unusual truncating PMM2 mutations in two CDG-la patients. Mol Genet Metab 2007; 90: 408-413.

7 Schollen E, Kjaergaard S, Legius E, Schwartz M, Matthijs G: Lack of Hardy-Weinberg equilibrium for the most prevalent PMM2 mutation in CDG-la (congenital disorders of glycosylation type la). Eur J Hum Genet 2000; 8: 367-371.

8 Grünewald S: The clinical spectrum of phosphomannomutase 2 deficiency (CDG-la). Biochim Biophys Acta 2009; 1792: 827-834.

9 Grünewald S, Schollen E, Van Schaftingen E, Jaeken J, Matthijs G: High residual activity of PMM2 in patients' fibroblasts: possible pitfall in the diagnosis of CDG-la (phosphomannomutase deficiency). Am J Hum Genet 2001; 68: 347-354.

10 Schollen E, Kjaergaard S, Martinsson T et al: Increased recurrence risk of congenital disorder of glycosylation type la (CDG-la) due to transmission ration distortion. J Med Genet 2004; 41: 877-880.

11 Dupré T, Cuer M, Barrot S et al: Congenital disorder of glycosylation la with deficient phosphomannomutase activity but normal plasma glycoprotein pattern. Clin Chem 2001; 47: 132-134.

12 Sturiale L, Barone R, Fiumara A et al: Hypoglycosylation with increased fucosylation and branching of serum transferrin N-glycans in untreated galactosemia. Glycobiology 2005; 15: 1268-1276.

13 Van Schaftingen E, Jaeken J: Phosphomannomutase deficiency is a cause of carbohydrate-deficient glycoprotein syndrome type I. FEBS Lett 1995; 377: 318-320.

14 Matthijs G, Schollen E, Cassiman JJ et al: Prenatal diagnosis in CDG1 families: beware of heterogeneity. Eur J Hum Genet 1998; 6: 99-104. 